\title{
Accurate DOA Estimation for Large-scale Uniform Circular Array Using a Single Snapshot
}

\author{
Qiang Li, Tao Su and Kai $\mathrm{Wu}$
}

\begin{abstract}
A large-scale antenna array is an enabling technique for millimeter wave communications. Uniform circular arrays (UCAs) have the spatial invariance property, ensuring the same beamforming performance in the whole angular region. However, the direction-of-arrival (DOA) estimation in UCAs is challenging, since the array response of a UCA does not conform to a Vandermonde structure as that of a uniform linear array (ULA). This paper proposes an accurate and low-complexity DOA estimation approach by exploiting the good correlation property of the array response of a UCA. The DOA estimates are first obtained from a circular convolution between a single snapshot and the designed coefficient vector. Then, by searching for the best initial phase of the coefficient vector, the DOA estimates can be refined to a configurable accuracy. Simulation results demonstrate that the proposed approach outperforms the state of the art by orders of magnitude in estimation accuracy.
\end{abstract}

Index Terms-Direction-of-arrival (DOA) estimation, array response vector, circular convolution

\section{INTRODUCTION}

$\mathbf{L}$ ARGE-SCALE uniform circular array (UCA) has been regarded as one of the most promising transceiver frontends in millimeter wave (mmWave) communications [1]. This is because the large number of antennas ensure the great beamforming gain, which can compensate for the severe path loss in mmWave bands [2]. On the other hand, a UCA has the spatial invariance property, i.e., the spatial resolution and pointing precision are invariant in the whole angular region $[0,2 \pi)$, making a UCA more desirable than a uniform linear array (ULA) to cover a wide angular region [3]. ${ }^{1}$

A critical issue to be addressed in UCAs is the accurate and efficient direction-of-arrival (DOA) estimation, which is the basis of beamforming, interference cancellation or localization [2], [4]. However, since the array response of a UCA does not conform to the Vandermonde form as a ULA, the DOA estimation in UCAs can therefore be more challenging than that in ULAs [3]. There has been up to date fewer studies on the efficient DOA estimatios in UCAs, as compared to ULAs.

The existing works on DOA estimation in UCAs were focused on using the conventional subspace-based DOA estimation techniques, e.g., UCA-MUSIC and UCA-ESPRIT [5]-

This work is partially supported by the National Natural Science Foundation of China (61671351), the National Science Fund for Distinguished Young Scholars (61525105), the Fund for Foreign Scholars in University Research and Teaching Programs (the 111 Project) (No. B18039).

Q. Li, T. Su (Corresponding author) and K. Wu are with the National Laboratory of Radar Signal Processing, Xidian University, Xi' an 710071, China (email: sirius.q.lee@outlook.com; sutao@xidian.edu.cn; ookaykay@163.com).

$\mathrm{K}$. Wu is also with the Global Big Data Technologies Centre (GBDTC), University of Technology Sydney (UTS), Sydney, NSW 2007, Australia.

${ }^{1}$ This is because a ULA has the wider beam width and increasingly reduced maximum beamforming gain as the beam is steered away from the array boresight.
[8]. In these methods, the array received signals were first transformed into phase mode and then the subspace-based techniques were applied based on the phase-mode representations of the received signals. However, the computational complexity of these methods can be prohibitive considering the huge amount of antennas in large-scale UCAs. This is because these methods require the eigenvalue decomposition, whose complexity grows cubically with the number of antennas [2].

In a different yet relevant context of DOA estimations in ULAs [9], an efficient estimation approach was developed by exploiting the Vandermonde structure of a ULA array response vector. Through the discrete Fourier transform (DFT) of a snapshot, coarse-resolution DOA estimates were obtained by enumerating the peaks of the DFT result. Then a phase rotation technique was developed to refine the above DOA estimates. As mentioned above, due to the dramatically different array structure, the approach is inapplicable for UCAs.

In this paper, we prove that the UCA array response vector has a good correlation property. Based on the finding, we propose to efficiently estimate the DOA through a circular convolution between the boresight UCA array response vector and a single snapshot. We also prove that in a certain angular region around an initial DOA estimate, the abovementioned circular convolution output has a single peak, which can be obtained through adjusting the initial phase of the match coefficient. Accordingly, we propose to refine the DOA estimates by searching for the optimal initial phase of the match coefficient around each estimate.

Other contributions also include the analysis on the computational complexity in comparison with the conventional subspace-based techniques [5], [6]. The Cramer-Rao lower bound of the obtained estimate is also derived with a closedform expression. Corroborated by numerical simulations, the proposed approach is able to outperform the state of the art [5], [6], [9] using only a single snapshot. It is also shown that UCAs are able to achieve much lower estimation errors than ULAs, which illustrates the superiority of UCAs.

The rest of the paper is organized as follows. In Section II, the system model is presented. The proposed DOA estimation approach is elaborated on in Section III. In Section IV, the computation complexity is analyzed and the CRLB of the DOA estimate is derived. Simulation results are provided in Section V, followed by conclusions in Section VI.

\section{SySTEM MODEL}

Fig.1 illustrates the large-scale UCA with $M(\gg 1)$ array elements, where the inter-element spacing is $d$, typically $d \leq \frac{\lambda}{2}$ [3]. $\lambda$ is the wavelength. Consider $K$ far-field sources with 


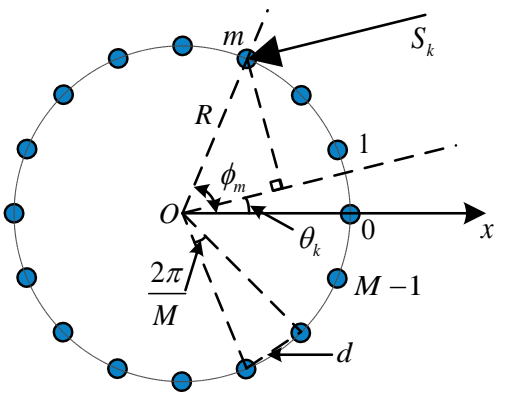

Fig. 1. Schematic illustration of a massive UCA.

DOAs collected by $\Theta=\left[\theta_{0}, \ldots, \theta_{K-1}\right]^{\mathrm{T}}$. $^{2}$ A single snapshot of the UCA, denoted by $\mathbf{x} \in \mathbb{C}^{M \times 1}$, can be written as

$$
\mathbf{x}=\mathbf{A s}+\mathbf{n},
$$

where $\mathbf{A}=\left[\mathbf{a}\left(\theta_{0}\right), \ldots, \mathbf{a}\left(\theta_{K-1}\right)\right]$ is the $M \times K$ array manifold matrix, $\mathbf{s}=\left[s_{0}, \ldots, s_{K-1}\right]^{\mathrm{T}}$ collects the $K \times 1$ information-bearing signals from the $K$ sources and $\mathbf{n} \in$ $\mathbb{C}^{M \times 1}$ is the additive white Gaussian noise (AWGN). $\mathbb{C}$ denotes the set of complex numbers.

$\mathbf{a}\left(\theta_{k}\right) \in \mathbb{C}^{M \times 1}$ is the array response at $\theta_{k}$, as given by $\mathbf{a}\left(\theta_{k}\right)=\left[\mathrm{e}^{\mathrm{j} 2 \pi \frac{R}{\lambda} \cos \left(\theta_{k}-\phi_{m}\right)}\right]_{m \in[0, M-1]}$, where $R$ is the radius of UCA, and $\phi_{m}=\frac{2 \pi m}{M}$ is the angle of the $m$-th array element with reference to the $x$-axis; refer to Fig.1. Based on the triangle relation in Fig.1, we have $2 R \sin \frac{\pi}{M}=d$. Therefore, $\mathbf{a}\left(\theta_{k}\right)$ can be simplified to

$$
\mathbf{a}\left(\theta_{k}\right)=\left[\mathrm{e}^{\mathrm{j} \varepsilon \cos \left(\theta_{k}-\phi_{m}\right)}\right]_{m \in[0, M-1]},
$$

where $\varepsilon=\frac{\pi d}{\lambda \sin \frac{\pi}{M}}$. The target of this letter is to estimate the DOA set $\Theta$ from the single-snapshot $\mathbf{x}$.

\section{ACCURATE AND FAST DOA ESTIMATION IN UCAS}

In this section, a two-step DOA estimation approach is designed. In the first step, we propose an efficient coarseresolution DOA estimation by exploiting the good correlation property of the UCA array response vectors. In the second step, the DOA estimates are refined by searching for the optimal initial phase that leads to the maximum array responses.

\section{A. Efficient DOA Search}

The key idea of the proposed efficient DOA search is to exploit the good correlation property of $\mathbf{a}\left(\theta_{k}\right)$, which is presented through the cross-correlation between $\mathbf{a}\left(\theta_{k}\right)$ and $\mathbf{a}^{*}\left(\phi_{l}\right) \cdot(\cdot)^{*}$ takes conjugate and we have $\phi_{l}=\frac{2 \pi l}{M}(l=$ $0,1, \ldots, M-1)$. For illustration convenience, we introduce $\mathbf{h}_{l}=\mathbf{a}^{*}\left(\phi_{l}\right)$. The cross-correlation between $\mathbf{h}_{l}$ and $\mathbf{a}\left(\theta_{k}\right)$ is defined as $r(l)=\mathbf{h}_{l}^{\mathrm{T}} \mathbf{a}\left(\theta_{k}\right)$, which has the following property.

Lemma 1: Provided a large $M(\gg 1),|r(l)| \ll\left|r\left(l^{\prime}\right)\right|$ for $l \in[0, M-1]$ and $l \neq l^{\prime}$, where $l^{\prime}=\left\lfloor\frac{M \theta_{k}}{2 \pi}\right\rceil$, i.e., the closest integer to $\frac{M \theta_{k}}{2 \pi}$.

Proof: See Appendix.

Fig. 2 plots $\frac{|r(l)|}{M}$ with $\frac{l}{M}$ as the abscissa, where $\theta_{k}=\frac{\pi}{2}$, and $M=64,128$ and 256 are taken to show the effect of $M$ on $\frac{|r(l)|}{M}$. By substituting the parameters into Lemma 1 , we

\footnotetext{
${ }^{2}$ For illustration convenience, we only consider the azimuth DOAs in this letter. The methods developed can be readily applied for elevation DOAs.
}

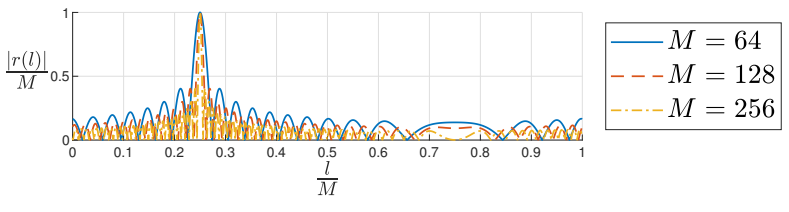

Fig. 2. Normalized $|r(l)|$ with different $M$.

can obtain that $l^{\prime}=16,32$ and 64 for $M=64,128$ and 256, respectively. We see from Fig. 2 that, when $\frac{l}{M}=\frac{1}{4},|r(l)|$ achieves the maximum, which verifies the above calculation of $l^{\prime}$. We also see that $|r(l)| \ll\left|r\left(l^{\prime}\right)\right|$ and, the larger $\left|l^{\prime}-l\right|$, the smaller $|r(l)|$ becomes, which validates Lemma 1.

In light of Lemma 1, we propose an efficient approach for DOA search as follows.

Proposition 1: The K DOA estimates can be searched efficiently through identifying the amplitude peaks of $\mathbf{y}=\mathbf{h}_{0} \otimes \mathbf{x}$, as given by

$$
\hat{\theta}_{k}^{\mathrm{c}}=\frac{2 \pi l_{k}^{\prime}}{M}, k=0,1, \cdots, K-1 .
$$

$|\mathbf{y}|$ achieves the peak at $l_{k}^{\prime}$ and $\otimes$ denotes circular convolution.

Proof: By suppressing the noise in (1), we obtain that

$$
\mathbf{y}=\mathbf{h}_{0} \otimes \mathbf{A} \mathbf{s}=\mathbf{h}_{0} \otimes \sum_{k=0}^{K-1} \mathbf{a}\left(\theta_{k}\right) s_{k}=\sum_{k=0}^{K-1} \mathbf{h}_{0} \otimes \mathbf{a}\left(\theta_{k}\right) s_{k},
$$

where the last equation is achieved due to the linear property of circular convolution [10]. (4) indicates that $\mathbf{y}$ is a linear combination of the convolutions $\mathbf{h}_{0} \otimes \mathbf{a}\left(\theta_{0}\right), \mathbf{h}_{0} \otimes \mathbf{a}\left(\theta_{1}\right), \cdots, \mathbf{h}_{0} \otimes$ $\mathbf{a}\left(\theta_{K-1}\right)$. The $l$-th element of $\mathbf{h}_{0} \otimes \mathbf{a}\left(\theta_{k}\right)$, denoted by $\mathbf{y}_{k}(l)$ $(l=0,1, \cdots, M-1)$, can be calculated as

$$
\mathbf{y}_{k}(l)=\mathbf{h}_{l}^{\mathrm{T}} \mathbf{a}\left(\theta_{k}\right)=r(l),
$$

where $\mathbf{h}_{l}$ is the $l$-shifted version of the flipped version of $\mathbf{h}_{0}$.

By exploiting Lemma $1, \mathbf{y}_{k}(l)$ achieves the peak at $l_{k}^{\prime}=$ $\left\lfloor\frac{M \theta_{k}}{2 \pi}\right\rceil$ for $k=0,1, \cdots, K-1$, and therefore (3) can be obtained. This concludes the proof.

Combining Lemma 1 and Proposition 1, we see that the $K$ DOA estimates in (3) have the maximum estimation error of $\frac{\pi}{M}$. This is because the search resolution in Proposition 1 is $\frac{2 \pi}{M}$, i.e., the sampling interval of $\mathbf{h}_{0}$ w.r.t. $m \in[0, M-1]$. In the following, we refine the DOA estimate by searching for the initial phase in a small angular region around $\hat{\theta}_{k}^{\mathrm{c}}$ with a much finer resolution, and, in turn, a much higher estimation accuracy of the DOA estimate can be achieved.

\section{B. Refinement of DOA Estimates}

Although the sampling interval of $\mathbf{h}_{0}$ w.r.t. $m \in[0, M-$ $1]$ is fixed given $M$, the phase of $\mathbf{h}_{0}$ can have an effect on the peak location of $\mathbf{y}_{k}(l)$. By adding an initial phase $\eta$ to $\mathbf{h}_{0}$, denoted by $\mathbf{h}_{0}^{\eta}=\mathbf{a}^{*}(\eta), \mathbf{y}_{k}(l)$ can be recalculated based on (5) and (10), as given in (6), where the same derivation techniques for (10) are used to obtain (6). We see from (6) that $\left|\mathbf{y}_{k}^{\eta}(l)\right|$ takes the maximum at $\theta_{k}=\left(\frac{2 \pi l}{M}+\eta\right)$. According to Proposition 1, $\left|\theta_{k}-\frac{2 \pi l_{k}^{\prime}}{M}\right| \leq \frac{\pi}{M}$, we can therefore refine $\hat{\theta}_{k}^{c}$ 


$$
\begin{aligned}
& \mathbf{y}_{k}^{\eta}(l)=\sum_{m=0}^{M-1} \mathrm{e}^{-\mathrm{j} \varepsilon \cos \left(\phi_{l-m}+\eta\right)} \mathrm{e}^{\mathrm{j} \varepsilon \cos \left(\theta_{k}-\phi_{m}\right)} \\
= & \sum_{m=0}^{M-1} \mathrm{e}^{-2 \mathrm{j} \varepsilon \sin \left[\frac{\theta_{k}}{2}+\frac{\pi(l-2 m)}{M}+\frac{\eta}{2}\right] \sin \left(\frac{\theta_{k}}{2}-\frac{\pi l}{M}-\frac{\eta}{2}\right)} \\
= & M \sum_{n=q M} \mathrm{j}^{n} J_{n}\left(2 \varepsilon \sin \left(\frac{\theta_{k}}{2}-\frac{\pi l}{M}-\frac{\eta}{2}\right)\right) \mathrm{e}^{\mathrm{j} n\left(\frac{\pi}{2}+\frac{\theta_{k}}{2}+\frac{\pi l}{M}+\frac{\eta}{2}\right)} \\
\approx & M J_{0}\left(2 \varepsilon \sin \frac{\left(\theta_{k}-\left(\frac{2 \pi l}{M}+\eta\right)\right)}{2}\right) .
\end{aligned}
$$

through searching for the $\eta$ leading to the maximum $\left|\mathbf{y}_{k}^{\eta}(l)\right|$, i.e.,

$$
\hat{\eta}_{k}=\arg \max _{\eta \in[-\pi / M, \pi / M]}\left|\mathbf{y}_{k}^{\eta}\left(l_{k}^{\prime}\right)\right|,
$$

where $\mathbf{y}_{k}^{\eta}\left(l_{k}^{\prime}\right)$ is obtained by substituting $l_{k}^{\prime}$ into (6). Therefore, the $K$ DOA estimates from Proposition 1 can be refined through the following proposition.

Proposition 2: Provided a large $M(\gg 1)$, the $k$-th $(k=$ $0,1, \cdots, K-1)$ DOA estimate, i.e., $\hat{\theta}_{k}^{\mathrm{c}}$ in (3), can be refined by substituting $l_{k}^{\prime}$ into (7) and searching for $\hat{\eta}_{k}$. The DOA estimate can be refined as $\hat{\theta}_{k}=\hat{\theta}_{k}^{\mathrm{c}}+\hat{\eta}_{k}$. The maximum estimation error is no greater than $\frac{\pi}{M G}$, where $G$ is the number of discrete angles in $\left[-\frac{\pi}{M}, \frac{\pi}{M}\right]$.

Proof: The proof of the proposition can be readily established based on (6) and (7), and therefore is suppressed for brevity.

Remark 1: Note that approximations are adopted in the proofs of Lemma 1 and Proposition 2. The approximations can be readily obtained based on a large $M(\gg 1)$. Take $M=128$ for an instance. By substituting $M=128$ into Appendix, we have $2 \varepsilon=\frac{\pi}{\sin \frac{\pi}{128}}=128.013$, and hence only an error of 0.013 is incurred by using the approximation of $\frac{\pi}{\sin \frac{\pi}{128}} \approx 128$. As such, we can check that $J_{2 M}(2 \varepsilon)=J_{256}(128.013)=$ $2.0096 \times 10^{-52}$ and $J_{3 M}(2 \varepsilon)=5.6193 \times 10^{-246}$, which validate the effectiveness of suppressing the high-order Bessel functions in (6) and (10).

\section{Performance AnAlysis \\ A. Analysis on Computational Complexity}

In this section, the computational complexity (in the number of complex multiplications) of the proposed approach is analyzed, in comparison to the conventional subspace-based approaches, e.g., UCA-MUSIC and UCA-ESPRIT [5].

The computational complexity of the proposed approach is dominated by the circular convolutions in (4) and (6), which can be given by $\mathcal{O}\left(2 M \log _{2}^{M}+M\right)$ and $\mathcal{O}(K G M)$, respectively. By exploiting the convolution theorem of the circular convolution ${ }^{3}$, (4) can be efficiently calculated by two DFT transforms and a pointwise product of two $M$ point sequences. For (6), $\mathcal{O}(K G M)$ is because $M$ complex multiplications are required for each DOA and sample of $\eta$.

The computational complexity of UCA-MUSIC or UCAESPRIT [5], is dominated by $\mathcal{O}\left(M^{2} L+M^{3}\right)$, where $\mathcal{O}\left(M^{2} L\right)$

\footnotetext{
${ }^{3}$ The convolution of two infinite sequences can be obtained as the inverse transform of the product of the individual transforms [10].
}

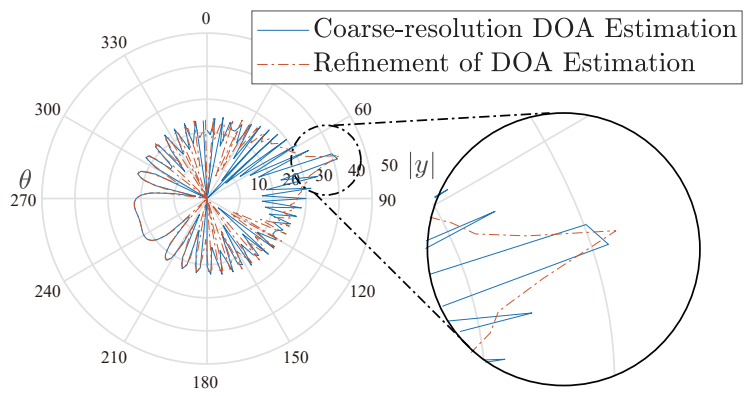

Fig. 3. Result of proposed method with $M=128, \theta=72^{\circ}, G=32$.

is for the calculation of the cross-correlation matrix based on $L$ snapshots, and $\mathcal{O}\left(M^{3}\right)$ is for the eigenvalue decomposition. We see that, in the case of large-scale UCAs, e.g., $M$ takes up to tens even hundreds, the computational complexity of UCAMUSIC or UCA-ESPRIT can be much larger than that of the proposed approach.

\section{B. CRLB of $\hat{\theta}_{k}$}

Let $\gamma_{k}$ denote the received SNR of the $k$-th source in (1). The CRLB of $\hat{\theta}_{k}$ can be given by [10]

$$
\operatorname{CRLB}\left(\hat{\theta}_{\mathrm{k}}\right)=\frac{1}{-\mathbb{E}\left[\frac{\partial^{2} \ln p(\mathbf{x} \mid \theta)}{\partial \theta^{2}}\right]}=\frac{1}{M \varepsilon^{2} \gamma_{k}},
$$

where $p(\mathbf{x} \mid \theta)$ is a likelihood function with $\theta$ as the parameter, and $\mathbf{x}$ is the observation vector. $\mathbb{E}$ denotes expectation and $\partial$ denotes partial derivative.

\section{Simulation Results}

In this section, simulation results are provided to validate the proposed approaches. Without loss of generality, we consider a UCA with $M=128$. The state-of-the-art UCA-RootMUSIC [7] is simulated as a benchmark, and the state-of-theart DOA estimation approach for ULA [9] is also simulated to demonstrate the superiority of UCA over ULA.

Fig. 3 illustrates the DOA estimates from the DOA search and refinement, respectively, where $K=1, \theta=72^{\circ}$ and $G=32$. We see that the peak of $|\mathbf{y}|$ is achieved at $l_{0}^{\prime}=$ 26 , and hence $\hat{\theta}_{0}^{\mathrm{c}}=73.13^{\circ}$, which validates Lemma 1 and Proposition 1. We also see that, by comparing $\left|\mathbf{y}_{0}^{\eta_{g}}\left(l_{0}^{\prime}\right)\right|$ for $\eta_{g}=-\frac{\pi}{M}+\frac{2 \pi g}{M G}(g=0,1, \cdots, G-1), \hat{\eta}_{0}$ can be taken at $g=3$. Therefore, the DOA estimate can be refined as $\hat{\theta}=$ $71.99^{\circ}$ with the estimation error of $|\hat{\theta}-\theta|=0.01^{\circ}$.

Fig. 4 compares the MSE of the DOA estimates using the proposed approach and the state-of-the-art approaches, where the two DOA $\theta_{1}$ and $\theta_{2}$ are independent of each other and uniformly distributed in the whole angular region, i.e., $[0,2 \pi)$. The number of snapshots required by the proposed approach and UCA-Root-MUSIC is 1 and 100, respectively. We see that the proposed approach outperforms UCA-Root-MUSIC dramatically and increasingly as SNR increases, even by using a much smaller number of snapshots. Particularly, in low SNR regions, we see that the average SNR improvement is larger than $5 \mathrm{~dB}$. We also see that the MSE of the proposed approach asymptotically reaches the CRLB with indistinguishable difference in high SNR regions, which further verifies the superiority of the proposed approach. 


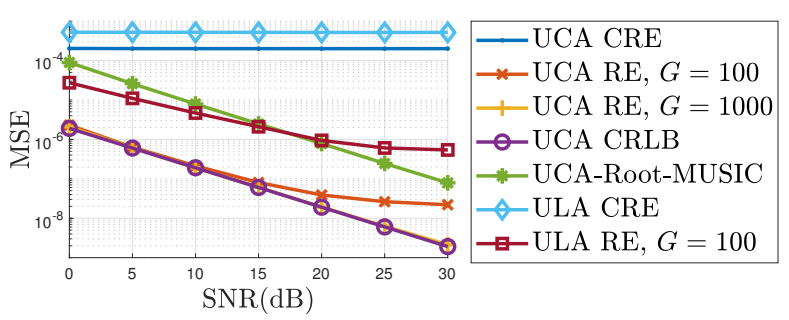

Fig. 4. MSE versus SNR with $M=128$, two sources, where "CRE" refers to "Coarse-Resolution Estimation" and "RE" refers to "Refinement Estimation".

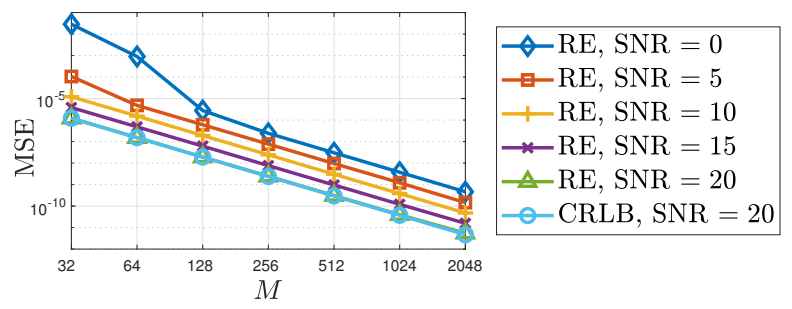

Fig. 5. MSE versus $M$ for UCA, two sources, and $G=1000$.

We also see from Fig. 4 that the proposed approach outperforms the state-of-the-art DOA estimation in ULAs. This is because UCA has the spatial invariance property, i.e., the steered beams have constant mainlobe widths in the whole angular region, $[0,2 \pi)$; whereas the beamwidth of the ULA increases with the pointing direction with reference to the normal direction of the array.

We also see from Fig. 4 that the proposed approach tends to converge in high SNR regions. This is because $G=100$ is taken in the simulation and hence the finest DOA refinement resolution is confined as $\frac{2 \pi}{G M}=4.9 \times 10^{-4}$. In turn, the maximum DOA refinement error can be $\left(\frac{4.9 \times 10^{-4}}{2}\right)^{2}=6.0239 \times$ $10^{-8}$. Nevertheless, this phenomenon can be suppressed by taking a larger $G$. According to the "CRLB UCA" in Fig. 4, we see that the analytical MSE of the proposed approach can be as low as $2 \times 10^{-9}$. By solving $\frac{\pi}{G M}=\sqrt{2 \times 10^{-9}}$, we obtain that $G \geq 549$ is required to have the MSE approaching the CRLB. To validate this this point, we further take $G=10^{3}$ and provide the simulated MSE versus SNR in Fig. 4. We see that the simulated MSE can indistinguishably approach the CRLB in the case of $G=10^{3}$.

Fig. 5 plots the MSE of the DOA estimate as $M$ increases, where different SNRs are taken. We see that, overall, the MSEs decreases gradually, as $M$ becomes larger. Particularly, in low SNR regions, e.g. $10 \mathrm{~dB}$, by increasing $M$ from 32 to 128 , the MSE reduces from $10^{-2}$ to $10^{-6}$. In addition, we see that the simulated MSE can indistinguishably approach the CRLB at $\mathrm{SNR}=20 \mathrm{~dB}$. This validates the effectiveness of the proposed approach.

\section{CONCLUSION}

In this letter, we propose a low-complexity DOA estimation method using a single snapshot, for massive UCA. With the good correlation property of array response, coarse-resolution DOA information can be extracted. Searching in the small region and changing the correlation coefficient, the refinement of DOA estimation can be performed. Compared to the traditional DOA algorithms for UCA, this method offers higher accuracy and lower complexity.

\section{APPENDIX}

Given $\mathbf{h}_{l}$ and $\mathbf{a}\left(\theta_{k}\right)$, we can calculate $r(l)$ as:

$$
r(l)=\sum_{m=0}^{M-1} \mathrm{e}^{-2 \mathrm{j} \varepsilon \sin \left[\frac{\theta_{k}}{2}+\frac{\pi(l-2 m)}{M}\right] \sin \left(\frac{\theta_{k}}{2}-\frac{\pi l}{M}\right),}
$$

where the angle difference identity of the cosine trigonometric function is used. By exploiting the Jacobi-Anger expansion, $\mathrm{e}^{\mathrm{j} \beta \cos \gamma}=\sum_{n=-\infty}^{+\infty} \mathrm{j}^{n} J_{n}(\beta) \mathrm{e}^{\mathrm{j} n \gamma}$ [3], $r(l)$ can be further calculated as

$$
\begin{aligned}
& r(l)=\sum_{n=-\infty}^{+\infty} \mathrm{j}^{n} J_{n}\left(2 \varepsilon \sin \left(\frac{\theta_{k}}{2}-\frac{\pi l}{M}\right)\right) \mathrm{e}^{\mathrm{j} n\left(\frac{\pi}{2}+\frac{\theta_{k}}{2}+\frac{\pi l}{M}\right)} \Xi \\
= & M \sum_{n=q M} \mathrm{j}^{n} J_{n}\left(2 \varepsilon \sin \left(\frac{\theta_{k}}{2}-\frac{\pi l}{M}\right)\right) \mathrm{e}^{\mathrm{j} n\left(\frac{\pi}{2}+\frac{\theta_{k}}{2}+\frac{\pi l}{M}\right)},(10)
\end{aligned}
$$

where $\Xi=\sum_{m=0}^{M-1} \mathrm{e}^{-\mathrm{j} 2 \pi \frac{n m}{M}}$ and $J_{n}(\beta)$ is the first-kind Bessel function of order $n$, and the last equality can be obtained based on the fact that

$$
\Xi=\left\{\begin{array}{lr}
M, & n=q M \\
0, & \text { otherwise }
\end{array}, \text { where } q \in \mathbb{Z} .\right.
$$

Note in (10) that $2 \varepsilon \sin \left(\frac{\theta_{k}}{2}-\frac{\pi l}{M}\right) \leq 2 \varepsilon=\frac{\pi}{\sin \frac{\pi}{M}} \approx M$, where the last approximation is due to $\sin \frac{\pi}{M} \cong \frac{\pi}{M}$ (given a large $M$ ). Therefore, $r(l)$ is only dominated by the components of $n=0, \pm M$, since $J_{n}\left(2 \varepsilon \sin \left(\frac{\theta_{k}}{2}-\frac{\pi l}{M}\right)\right) \approx 0$ for $n \geq 2 M$. Furthermore, in the considered massive UCA, we have $M \gg 1$ and hence the maximum amplitude of $J_{M}\left(2 \varepsilon \sin \left(\frac{\theta_{k}}{2}-\frac{\pi l}{M}\right)\right) \approx J_{M}(M)$ is negligible compared with the maximum amplitude of $J_{0}\left(2 \varepsilon \sin \left(\frac{\theta_{k}}{2}-\frac{\pi l}{M}\right)\right) \approx$ $J_{0}(0)=1$. This indicates that, provided $M$ is large, $r(l)$ can be approximated solely by the sinc-like function $J_{0}\left(2 \varepsilon \sin \left(\frac{\theta_{k}}{2}-\frac{\pi l}{M}\right)\right)$. Therefore, when $\left|\frac{\theta_{k}}{2}-\frac{\pi l}{M}\right|$ approaches $0, r(l)$ achieves the maximum, leading to $l=\left\lfloor\frac{M \theta_{k}}{2 \pi}\right\rceil$.

\section{REFERENCES}

[1] J. Zhang et al., "5G Millimeter-Wave Antenna Array: Design and Challenges," IEEE Wireless Commun., vol. 24, no. 2, pp. 106-112, April 2017.

[2] K. Wu et al., "Robust Unambiguous Estimation of Angle-of-Arrival in Hybrid Array With Localized Analog Subarrays," IEEE Trans. Wireless Commun., vol. 17, no. 5, pp. 2987-3002, May 2018.

[3] S. C. Chan et al., "Uniform Concentric Circular Arrays With FrequencyInvariant Characteristics-Theory, Design, Adaptive Beamforming and DOA Estimation," IEEE Transactions on Signal Processing, vol. 55, no. 1, pp. 165-177, Jan 2007.

[4] K. Wu et al., "Fast and Accurate Estimation of Angle-of-Arrival for Satellite-Borne Wideband Communication System," IEEE J. Sel. Areas in Commun., vol. 36, no. 2, pp. 314-326, Feb 2018.

[5] C. P. Mathews et al., "Eigenstructure techniques for 2-D angle estimation with uniform circular arrays," IEEE Trans. Signal Process., vol. 42, no. 9, pp. 2395-2407, Sep 1994.

[6] _ , "Performance analysis of the UCA-ESPRIT algorithm for circular ring arrays," IEEE Trans. Signal Process., vol. 42, no. 9, pp. 2535-2539, Sep 1994.

[7] G. Jiang et al., "Reducing errors for root-MUSIC-based methods in uniform circular arrays," IET Signal Processing, vol. 12, no. 1, pp. 3136, 2018.

[8] R. Goossens et al., "UCA Root-MUSIC With Sparse Uniform Circular Arrays," IEEE Trans. Signal Process., vol. 56, no. 8, pp. 4095-4099, Aug 2008

[9] R. Cao et al., "A Low-Complex One-Snapshot DOA Estimation Algorithm with Massive ULA," IEEE Commun. Lett., vol. 21, no. 5, pp. 1071-1074, May 2017.

[10] H. L. V. Trees, Detection, Estimation and Modulation Theory. New York: Wiley, 1968. 